\title{
Photoacoustic Super-Resolution Imaging using Laser Activation of Low-Boiling-Point Dye-Coated Nanodroplets in vitro and in vivo
}

\author{
Ge Zhang ${ }^{1}$, Sevan Harput ${ }^{1}$, Anant Shah ${ }^{2}$, Javier Hernández-Gil ${ }^{3}$, Jiaqi Zhu ${ }^{1}$, Kirsten Christensen-Jeffries ${ }^{4}$, Jemma \\ Brown ${ }^{4}$, Nicholas J. Long ${ }^{3}$, Robert J. Eckersley ${ }^{4}$, Chris Dunsby ${ }^{5}$, Jeffrey Bamber ${ }^{2}$, Meng-Xing Tang ${ }^{1}$ \\ ${ }^{1}$ Department of Bioengineering, Imperial College London, London, United Kingdom \\ 2 Joint Department of Physics and CRUK Cancer Imaging Centre, The Institute of Cancer Research and The Royal Marsden NHS \\ Foundation Trust, London, United Kingdom \\ ${ }^{3}$ Department of Chemistry, Imperial College London, London, United Kingdom \\ ${ }^{4}$ Biomedical Engineering Department, School of Biomedical Engineering and Imaging Sciences, Kings College London, London, \\ United Kingdom \\ ${ }^{5}$ Department of Physics and Centre for Pathology, Imperial College London, London, United Kingdom \\ Email: Mengxing.Tang@Imperial.ac.uk
}

\begin{abstract}
In this study, a photoacoustic super-resolution imaging technique was developed through imaging the activation of Cyanine 7.5-coated phase-change nanodroplets using a preclinical photoacoustic imaging system and localizing the activated droplets. As a proof-of-concept experiment, photoacoustic images of flowing dye-coated nanodroplets in microfluidic channels were obtained with a cylindrically focused curved-array. Experimental results showed that super-resolution images can resolve the microfluidic channels which cannot be resolved by conventional beamformed images. The results also showed that the dye-coated phase-change nanodroplets can be optically activated in vivo and the activation signals can be separated from the image background by applying singular value decomposition filtering, and be used for further superlocalization processing. Such nanodroplets can offer better biocompatibility, as well as more flexible and controllable droplet activation rates, with potential for super-resolution imaging of static and extravascular targets, compared to existing contrast agents used in existing localization-based photoacoustic superresolution imaging techniques.
\end{abstract}

Keywords - Super-Resolution, Photoacoustics, Nanodroplets, MSOT System, Laser

\section{INTRODUCTION}

Photoacoustic imaging is a biomedical imaging modality which can provide high-resolution, multi-contrast images of biological structures [1]. Absorption of light by endogenous biomolecules or exogenous contrast agents, thermoelastically induces acoustic waves, the detection of which is used to form an image [2]. However, when diffuse illumination is used, the spatial resolution of photoacoustic imaging is inherently limited by the acoustic detection bandwidth [3].
Over the last decade, various optical super-resolution imaging techniques have been developed to break the diffraction limit in spatial resolution, and photo-activated localization microscopy (PALM) [4] and stochastic reconstruction microscopy (STORM) [5] are examples of localization methods based on sequentially acquired image data. Such methods are capable of resolving cellular structures at spatial resolutions of a few tens of nanometers. Inspired by optical super-resolution imaging, ultrasound super-resolution imaging techniques using flowing microbubble contrast agents were developed in order to resolve microvascular structures beyond the diffraction limit in deeper regions of tissues [6,7]. High-boiling-point perfluorocarbon nanodroplets have been used in photoacoustic super-resolution imaging as they can be repeatedly activated by laser pulses between which they recondense to continuously provide localization events [8]. Another study demonstrated that high-boiling-point perfluorocarbon nanodroplets can be used for subwavelengthlocalized ultrasound-assisted drug delivery [9]. Recently, our group developed a new ultrasound super-resolution imaging approach using acoustic activation and deactivation of lowboiling-point nanodroplet contrast agents, which we called acoustic wave sparsely activated localization microscopy (AWSALM) [10]. Unlike ultrasound super-resolution imaging using microbubbles, AWSALM does not require flow to generate new super-resolution signals for super-resolved localization of microbubbles over time. It also requires much less energy/pressure to perform super-resolution imaging compared to other droplet-vaporization based super-resolution techniques. In the photoacoustics community, deep-tissue photoacoustic super-resolution imaging was first investigated without contrast agents, using optical wavefront shaping approaches, to allow resolution to be optically determined rather than acoustically determined [11]. Inspired by superresolution optical fluctuation imaging (SOFI) [12], non- 
contrast statistical methods apply high-order statistics of deliberately varied illumination speckle to achieve photoacoustic super-resolution [13, 14]. Contrast particlelocalization based super-resolution photoacoustic imaging was first developed using flowing optically absorbing polyethylene microspheres $[3,15]$.

We have previously demonstrated that cyanine 7.5-coated nanodroplets, termed as 'Cy-droplets', can be used to enhance contrast in photoacoustic imaging, generating a $56 \mathrm{~dB}$ increase in signal strength relative to controls such as microbubbles, Cy-microbubbles and Cy-solution [16]. Cy-droplets consist of a low-boiling-point perfluorocarbon core for low-threshold optical activation, and an optically absorbing dye chosen to absorb light at a wavelength in the near-infrared range with good tissue penetration. Nanodroplets containing a lowboiling-point perfluorocarbon are easier to activate and their use is thus less likely to be associated with unwanted ultrasound bioeffects than those with a high-boiling-point core, especially when activating in deeper tissues [17-19].

Compared to the existing localization-based photoacoustic super-resolution imaging techniques using optically absorbing paramagnetic polyethylene microspheres, nanodroplet contrast agents offer better biocompatibility and clearance from living systems. Moreover, the use of dye-coated nanodroplets provides a more controllable super-resolution imaging environment. This is because the concentration of microspheres cannot be changed once injected, whereas the activation of nanodroplets is governed by laser intensity, wavelength, and the concentration of nanodroplets. This means, the sparsity of droplet activation signals can be adjusted in real-time by manipulating the laser intensity and wavelength after the injection. Furthermore since, unlike absorbing microspheres, the photoacoustic signals from Cy-droplets are generated at the moment of activation, and they should be able to extravasate due to enhanced permeability and retention effect [20-22], super-resolution imaging with the Cy-droplets need not require flow nor be restricted to visualizing the microvasculature.

Inspired by AWSALM and the above properties of Cydroplets, we have developed a photoacoustic super-resolution imaging technique, and this is demonstrated here using laser activation of Cy-droplets in vitro. We also show the activation patterns of Cy-droplets in vivo, which demonstrates the potential of applying this technique in vivo.

\section{METHODS AND EXPERIMENT}

\section{A. Nanodroplet Preparation}

The Cy-droplets were fabricated as described previously $[16][\underline{23}, 24]$. Briefly, the lipid shell consists of 1,2dipalmitoyl-sn-glycero-3-phosphocholine (DPPC), 1,2dipalmitoyl-sn-glycero-3-phosphoethanolamine-N-

[methoxy(polyethylene glycol)-2000](16:0 PEG2000 PE) and DSPE-PEG(2000)-Cyanine 7.5 9:0.8:0.2, m:m:m (total lipid concentration of $0.85 \mathrm{mg} / \mathrm{mL}$ ) dissolved in a solution of propylene glycol, glycerol, and phosphate-buffered saline (PBS) $(15 / 5 / 8, v / v / v)$. The resulting lipid solution was sealed in a glass vial and the headspace was then purged with decadluorobutane gas at room temperature. The precursor Cymicrobubbles were generated by mechanical agitation. The Cydroplet emulsion was obtained by pressurization and condensation using the method detailed in [25]. The Cy-droplet solution was diluted to $10 \%$ relative to stock solution in the distilled/deionized water [트, 26, 27] .

\section{B. Experimental Setup}

A schematic of the experimental setup used is shown in Fig. 1. The phantom was made of two microfluidic cellulose channels (diameter $200 \mu \mathrm{m}$ and $300 \mu \mathrm{m}$ respectively, wall thickness $8 \pm 1 \mu \mathrm{m}$ in the dry state, Hemophan ${ }^{\circledR}$, Membrana) embedded in $1.5 \%$ agarose gel. The channels were embedded into the agarose phantom with a diameter of $3 \mathrm{~cm}$.

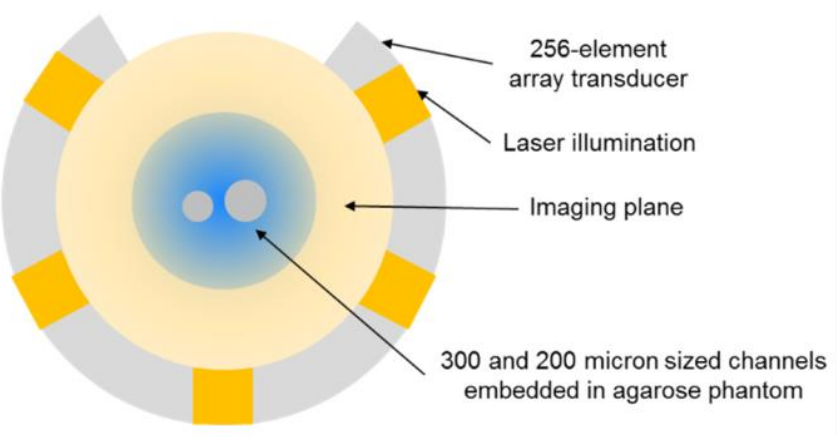

Fig. 1. Experimental setup. Photoacoustic signals were measured with 256 elements distributed over $270^{\circ}$ of a cylindrically focused curved-array (radius $40 \mathrm{~mm}$, nominal peak sensitivity at $5 \mathrm{MHz}$, element pitch $0.735 \mathrm{~mm}$ ) within an MSOT in Vision 256-TF ${ }^{\mathrm{TM}}$ (iThera Medical $\mathrm{GmbH}$ ). The yellow plane indicates the imaging plane and the transducer channels are oriented perpendicular to the plane of the figure.

C. Image Acquisition

Photoacoustic imaging was performed at a single wavelength of $788 \mathrm{~nm}$ (peak absorption of Cyanine 7.5) with a fluence per pulse estimated at the surface of the mouse body to be $20 \mathrm{~mJ} / \mathrm{cm}^{2}$, based on manufacture-nominal total laser energy per pulse and the approximate surface area of a mouse illuminated. One pulse $(10 \mathrm{~ns}$ pulses, $10 \mathrm{~Hz}$ pulse repetition frequency) was transmitted per image. The raw data was beamformed by the back-projection algorithm embedded in the MSOT system (viewMSOT v3.8) in order to obtain the image data.

\section{Image Analysis}

Super-localization processing was then applied in order to localize the activation signals of the nanodroplets. For the reconstruction of the super-resolution image, a minimum image pixel value threshold was set in order to reject noise and detect potential droplet activation. After thresholding, each detected potential event in each image frame was compared to the point-spread-function (PSF) of the imaging system according to its intensity, area, and shape. Events that did not fit the properties of a single PSF are assumed to be likely due 
to either simultaneous activation of multiple neighboring nanodroplets or noise $[\underline{10}, \underline{28}]$. These events were discarded after the comparisons. The location of each single isolated droplet activation signal was then calculated by the "centroid method' []. The centroid of each localized signal was computed by estimating the intensity weighted center of mass. The final super-resolution image was created from accumulating all the localizations detected over all the imaging frames.

\section{REASULTS AND DISCUSSIONS}

(a) Conventional Image

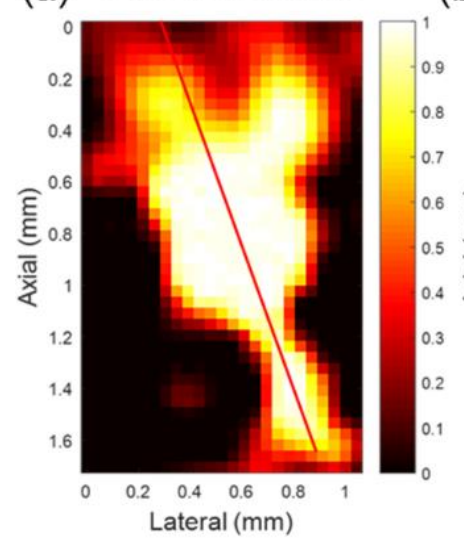

(b) Super-Resolution Image

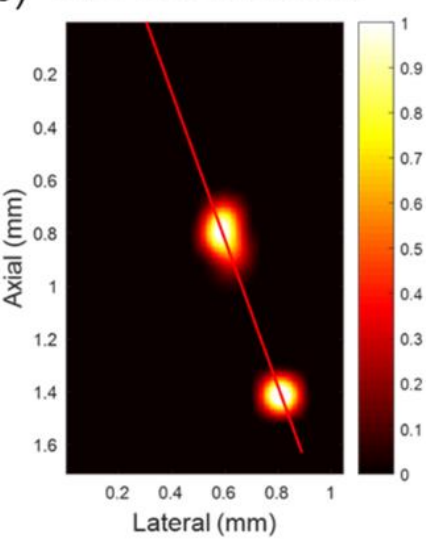

(c)

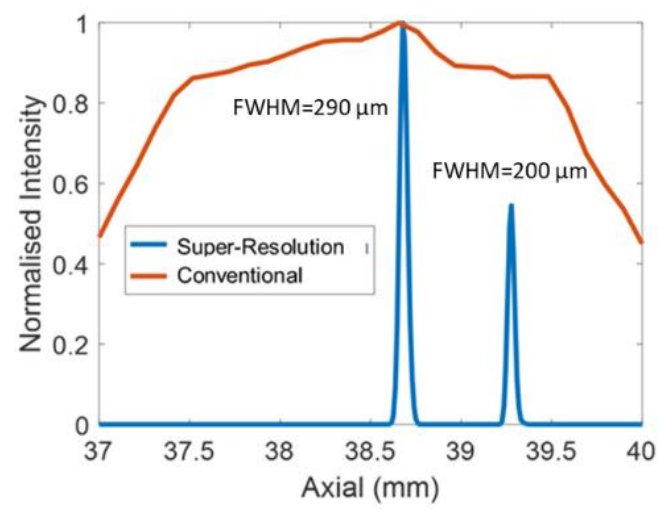

Fig. 2. Experimental results. (a) Summation of 500 conventional crosssectional photoacoustic images of the cross-section of the micro-channels. The two neighboring channels cannot be resolved. (b) Super-resolution photoacoustic image of the micro-channel cross-sections. (c) Profile through conventional and super-resolution images along the red lines indicated on (a) and (b).

Fig. 2(a) shows the summation of 500 conventional beamformed images of the cross-section of the tube phantom, acquired in 50 seconds. Fig. 3(b) shows the corresponding super-resolution image. It can be seen from Fig. 3(c) that the super-resolution image can resolve two tubes whereas the original beamformed image cannot. Moreover, the full-widthhalf-maximum (FWHM) of the two peaks in the superresolution image were measured to be 290 and $200 \mu \mathrm{m}$ for the 300 and $200 \mu \mathrm{m}$ diameter tubes respectively.
The main limitation of this initial proof-of-concept study is that the pulse repetition frequency of the laser in the MSOT system used is $10 \mathrm{~Hz}$, which limits the in vivo data that can be acquired. Thus, an unacceptably long acquisition time would be required to accumulate sufficient localization events for reconstructing a super-resolution in vivo image. Therefore, the current preclinical imaging system has limited in vivo usefulness given the allowable times for animal anaesthesia. The low pulse repetition rate also, prevents the observation of dynamic events and would compromise the spatial resolution unless adequate motion correction can be applied. This limitation was also present in other localization-based photoacoustic super-resolution imaging studies [3, 15]. Nevertheless, if it can be overcome through the use of higher repetition rate laser sources, localization-based superresolution imaging provides a very simple and promising way to improve the resolution. It should be noted that the curved array transducer in this study was used to demonstrate this technique. Future clinical studies can be performed using array transducers of different geometries including linear array currently used in clinical ultrasound. The imaging depth will always be limited by the penetration depth of the laser, regardless of the transducer geometry used.

The apparent stochasticity of signals may come from the droplet vaporization process due to a range of factors. One important factor is the droplet population consists of droplets with a statistical distribution of different size and shell properties. These Cy-droplets are polydisperse with sizes ranging from $400-600 \mathrm{~nm}$ [16]. The droplet size is an important factor determining the activation threshold and it was found that the activation threshold is inversely proportional to the droplet diameter [17]. Therefore, only a sparse population of large droplets can be activated during the first laser pulse. The droplets activated by the next laser pulse may be affected by a number of factors. First, it may come from photobleaching of the encapsulated dye and splitting or coalescing of droplets during vaporization [8]. Second, they may be affected by any increase in local temperature due to the absorption of laser energy, as droplets have a lower activation pressure as temperature increases [29]. Finally, in the case of flow imaging, the motion of the droplets adds another level of stochasticity.

As for the biocompatibility and translatability of the lipid and perfluorocarbon, the homemade Cy-droplets solution has the same core composition as the commercial MicroMarker(C) (FUJIFILM, Visualsonics) and SonazoidC (GE Healthcare) contrast microbubbles. A previous study has shown that the commercial MicroMarker(C) microbubble contrast agents can be condensed into nanodroplets [30]. As for the dye lipid, it should be noted that $\mathrm{Cy}-7.5$ has not been approved by the FDA. Therefore, nanodroplets coated with indocyanine green 
[31], which is already approved by the FDA, can be manufactured for the future clinical investigation. These could potentially facilitate the clinical translation of this photoacoustic super-resolution imaging technique.

\section{CONCLUSION}

In conclusion, a photoacoustic super-resolution imaging technique was developed and demonstrated using the activation of Cyanine-7.5 coated nanodroplets generated in vitro by a preclinical photoacoustic system. The experimental results showed that the super-resolution image can resolve structures which cannot be resolved by the conventional beamformed image. We have also shown that it is possible to activate the Cyanine-7.5 coated nanodroplets in vivo and that the activation signals can be localized. Compared to contrast agents used in previous localization-based photoacoustic super-resolution imaging research, dye-coated nanodroplets offer better biocompatibility, the potential for imaging extravascular structure which may be reached by molecularlytargeted versions of the nanodroplets, and potential for imaging in the absence of flow. This is also the first demonstration that any time-resolved localizable photoacoustic contrast signals can be detected in vivo.

\section{ACKNOWLEDGMENT}

This work was supported by the EPSRC under Grant EP/N015487/1 and EP/N014855/1, EP/L015226/1, WT 203148/Z/16/Z. This work is also supported by the CRUK Multidisciplinary Project Award (No. C53470/A22353), and Imperial-ICR CRCE pump-priming awards.

\section{REFERENCES}

1. Beard, P., Biomedical photoacoustic imaging. Interface Focus, 2011. 1(4): p. 602-631.

2. Wang, L.V. and S. Hu, Photoacoustic Tomography: In Vivo Imaging from Organelles to Organs. Science, 2012. 335(6075): p. 1458-1462.

3. Vilov, S., B. Arnal, and E. Bossy, Overcoming the acoustic diffraction limit in photoacoustic imaging by the localization of flowing absorbers. Optics Letters, 2017. 42(21): p. 4379-4382.

4. Betzig, E., et al., Imaging Intracellular Fluorescent Proteins at Nanometer Resolution. Science, 2006. 313: p. 1643-1645.

5. Rust, M.J., M. Bates, and X. Zhuang, Sub-diffraction-limit imaging by stochastic optical reconstruction microscopy (STORM). Nature Methods, 2006. 3: p. 793.

6. Christensen-Jeffries, K., et al., In Vivo Acoustic Super-Resolution and Super-Resolved Velocity Mapping Using Microbubbles. Ieee Transactions on Medical Imaging, 2015. 34(2): p. 433-440.

7. Errico, C., et al., Ultrafast ultrasound localization microscopy for deep super-resolution vascular imaging. Nature, 2015. 527(7579): p. 499-502.

8. Luke, G.P., A.S. Hannah, and S.Y. Emelianov, Super-Resolution Ultrasound Imaging in Vivo with Transient Laser-Activated Nanodroplets. Nano Lett, 2016. 16(4): p. 2556-9.

9. Hingot, V., et al., Subwavelength far-field ultrasound drug-delivery. Applied Physics Letters, 2016. 109(19): p. 194102.

10. Zhang, G., et al., Acoustic wave sparsely activated localization microscopy (AWSALM): Super-resolution ultrasound imaging using acoustic activation and deactivation of nanodroplets. Applied Physics Letters, 2018. 113(1): p. 014101.

11. Conkey, D.B., et al., Super-resolution photoacoustic imaging through a scattering wall. Nature Communications, 2015. 6: p. 7902.
12. Dertinger, T., et al., Superresolution Optical Fluctuation Imaging (SOFI), in Nano-Biotechnology for Biomedical and Diagnostic Research, E. Zahavy, et al., Editors. 2012, Springer Netherlands: Dordrecht. p. 17-21.

13. Hojman, E., et al., Photoacoustic imaging beyond the acoustic diffraction-limit with dynamic speckle illumination and sparse joint support recovery. Optics Express, 2017. 25(5): p. 4875-4886.

14. Chaigne, T., et al., Super-resolution photoacoustic fluctuation imaging with multiple speckle illumination. Optica, 2016. 3(1): p. 54-57.

15. Luís Dean-Ben, X. and D. Razansky, Localization optoacoustic tomography. Light: Science \&Amp; Applications, 2018. 7: p. 18004.

16. Lin, S., et al., Optically and acoustically triggerable sub-micron phasechange contrast agents for enhanced photoacoustic and ultrasound imaging. Photoacoustics, 2017. 6: p. 26-36.

17. Sheeran, P.S., et al., Decafluorobutane as a Phase-Change Contrast Agent for Low-Energy Extravascular Ultrasonic Imaging. Ultrasound in Medicine and Biology, 2011. 37(9): p. 1518-1530.

18. Zhang, G., et al. Acoustic response of targeted nanodroplets postactivation using high frame rate imaging. in 2017 IEEE International Ultrasonics Symposium (IUS). 2017.

19. Zhang, G., et al. Acoustic Wave Sparsely-Activated Localization Microscopy (AWSALM): A Fast and Flow Independent Ultrasound Super-Resolution Technique using Nanodroplets. in 2018 IEEE International Ultrasonics Symposium (IUS). 2018.

20. Mannaris, C., et al., Microbubbles, Nanodroplets and Gas-Stabilizing Solid Particles for Ultrasound-Mediated Extravasation of Unencapsulated Drugs: An Exposure Parameter Optimization Study. Ultrasound in Medicine \& Biology, 2019. 45(4): p. 954-967.

21. Zhang, G., et al. Investigation of Nanodroplet Adhesion to Endothelial Cells Under Atheroprone Flow Conditions. in 2018 IEEE International Ultrasonics Symposium (IUS). 2018.

22. Zhang, G., et al. Super-Localisation Ultrasound Imaging using Sparse Activation of Low-Boiling-Point Nanodroplets. in The 23rd European Symposium on Ultrasound Contrast Imaging. 2018. Rotterdam, Netherlands.

23. Zhang, G., et al. Acoustic Wave Sparsely-Activated Localization Microscopy (AWSALM): In Vivo Fast Ultrasound Super-Resolution Imaging using Nanodroplets. in 2019 IEEE International Ultrasonics Symposium (IUS). 2019.

24. Zhang, G., et al. Minimization of Nanodroplet Activation Time using Focused-Pulses for Droplet-Based Ultrasound Super-Resolution Imaging. in 2019 IEEE International Ultrasonics Symposium (IUS). 2019.

25. Zhang, G., et al., Quantification of Vaporised Targeted Nanodroplets Using High-Frame-Rate Ultrasound and Optics. Ultrasound in Medicine \& Biology, 2019. 45(5): p. 1131-1142.

26. Zhang, G., et al. Contrast-Enhanced Photoacoustic Imaging of Lowboiling-point Phase-Change Nanodroplets. in 2019 IEEE International Ultrasonics Symposium (IUS). 2019.

27. Zhang, G., et al. High Signal-to-Noise Ratio Contrast-Enhanced Photoacoustic Imaging using Acoustic Sub-Aperture Processing and Spatiotemporal Filtering. in 2019 IEEE International Ultrasonics Symposium (IUS). 2019.

28. Zhang, G., et al., Fast Acoustic Wave Sparsely Activated Localization Microscopy (fast-AWSALM): Ultrasound Super-Resolution using PlaneWave Activation of Nanodroplets. IEEE Transactions on Ultrasonics, Ferroelectrics, and Frequency Control, 2019: p. 1-1.

29. Sheeran, P.S. and P.A. Dayton, Improving the performance of phasechange perfluorocarbon droplets for medical ultrasonography: current progress, challenges, and prospects. Scientifica (Cairo), 2014. 2014: p. 579684

30. Sheeran, P.S., et al., More Than Bubbles: Creating Phase-Shift Droplets from Commercially Available Ultrasound Contrast Agents. Ultrasound Med Biol, 2016.

31. Hannah, A., et al., Indocyanine green-loaded photoacoustic nanodroplets: dual contrast nanoconstructs for enhanced photoacoustic and ultrasound imaging. ACS nano, 2014. 8(1): p. 250-259. 